Georgian Mathematical Journal

Volume 13 (2006), Number 4, 807-812

\title{
COPRIMENESS AMONG ELEMENT ORDERS OF FINITE GROUPS
}

\author{
XINGZHONG YOU
}

\begin{abstract}
In this paper we classify the finite groups satisfying the following property $P_{3}$ : every three distinct orders of elements are setwise relatively prime.
\end{abstract}

2000 Mathematics Subject Classification: 20D60, $20 \mathrm{E} 34$.

Key words and phrases: Finite group, element order, prime graph, simple group.

1. Introduction. Let $G$ be a finite group and $C h(G)$ be one of the following sets:

$c d(G)=\{\chi(1) \mid \chi \in \operatorname{Irr}(G)\}$, i.e. the set of irreducible character degrees of $G$;

$\operatorname{con}(G)=\left\{g^{G} \mid g \in G\right\}$, i.e., the set of conjugacy classes of $G$; and

$\pi_{e}(G)=\{o(g) \mid g \in G\}$, i.e., the set of element orders of $G$.

We say that $G$ has the following property $P_{n}$ : if $C h(G)=c d(G)$ or $\pi_{e}(G)$ then every set of $n$ distinct elements of $C h(G)$ is setwise coprime; if $C h(G)=\operatorname{con}(G)$ then their sizes are setwise coprime for any $n$ distinct elements of $C h(G)$.

In [1], the author studied the structure of the solvable group $G$ with $P_{n}$ when $C h(G)=c d(G)$, where a quadratic bound that depends only on $n$ for $|c d(G)|$ was obtained. It was conjectured in [2] that if any prime integer $p$ divides at most $m$ elements in $c d(G)$, then $|c d(G)| \leq 3 m$. In [3], the authors studied the structure of the group $G$ with $P_{n}$ when $C h(G)=\operatorname{con}(G)$. In particular, they classified the finite groups when $n=5$, extending the result in [4].

In this paper, we study the structure $G$ with $P_{n}$ in the case of $C h(G)=\pi_{e}(G)$. We classify the finite groups that satisfy property $P_{3}$, extending the results in [5] and [6]. We also obtain a bound that depends only on $n$ for $|\pi(G)|$, the number of distinct prime divisors of the order of $G$.

2. Preliminaries. In the following we always assume that property $P_{n}$ takes place in the case $C h(G)=\pi_{e}(G)$. First, we present some preliminary results we need.

Lemma 2.1. Let $G$ be a finite group that satisfies property $P_{n}$. Then property $P_{n}$ is inherited by subgroups and quotient groups of $G$.

Proof. The proof is straightforward.

Lemma 2.2. Let $r$ be a prime and $n$ a positive integer such that $\frac{q+1}{d}$ and $\frac{q-1}{d}$ are products of at most two primes, where $q=r^{n}$ and $d=(r-1,2)$.

(a) If $r>2$ and $q \equiv 3$ or $5(\bmod 8)$, then $n=1$ or an odd prime. 
(b) If $r=2$, then $n=1,2$ or an odd prime.

Proof. (a) Suppose that $r>2$. If $q \equiv 3(\bmod 8)$, then $4 \not \nmid q-1$. If $n=2 m$, then $q-1=\left(r^{m}+1\right)\left(r^{m}-1\right)$ and so that $4 \mid q-1$, a contradiction; if $n=k m$ is odd with $k, m>1$, then $q^{n}-1=(q-1)\left(q^{m-1}+q^{m-2} \cdots+q+1\right)\left(q^{m(k-1)}+\right.$ $\left.q^{m(k-2)}+\cdots+q^{m}+1\right)$, a contradiction. Using a similar argument, we also conclude that if $q \equiv 5(\bmod 8)$, then $n=1$ or an odd prime.

(b) Suppose that $r=2$. If $n=4 m$, then $2^{n}-1=\left(2^{2 m}+1\right)\left(2^{m}+1\right)\left(2^{m}-1\right)$, a contradiction; if $n=4 m+2$, then $2^{n}-1=\left(2^{2 m+1}-1\right)(2+1)\left(2^{2 m}-2^{2 m-1}+\right.$ $\left.2^{2 m-2}-\cdots+2^{2}-2+1\right)$, a contradiction; if $n=k m$ is odd with $k, m>1$, then $2^{n}+1=(2+1)\left(2^{k-1}-2^{k-2}+2^{k-3}-\cdots+2^{2}-2+1\right)\left(2^{k(m-1)}-2^{k(m-2)}+\right.$ $\left.2^{k(m-3)}-\cdots+2^{2 k}-2^{k}+1\right)$, a contradiction.

Recall that the prime graph $\Gamma(G)$ of $G$ is defined as follows: its vertex set is $\pi(G)$, and two distinct vertices $p, q$ in $\pi(G)$ are connected with an edge if $p q \in \pi_{e}(G)$. Denote by $t(G)$ the number of connected components of $\Gamma(G)$ and by $\pi_{i}=\pi_{i}(G), i=1,2, \ldots, t(G)$ the connected components of $\Gamma(G)$. If $G$ is of even order, we always assume that $2 \in \pi_{1}$.

A group $G$ is 2-Frobenius if there exists a normal series $1 \triangleleft N \triangleleft K \triangleleft G$ such that $G / N$ and $K$ are Frobenius groups with kernels $K / N$ and $N$ respectively. For any 2-Frobenius $G$, we have $t(G)=2$ and $\pi_{1}=\pi(N) \cup \pi(G / K), \pi_{2}=\pi(K / N)$. In particular, $G$ is solvable.

In relation to the number of connected components of $\Gamma(G)$ we have the following Lemma due to Gruenberg and Kegel (see Theorem A [7]).

Lemma 2.3. Let $G$ be a finite group with disconnected prime graph, then $G$ has one of the following structures:

(a) Frobenius or 2-Frobenius;

(b) simple;

(c) an extension of a $\pi_{1}$-group by a simple group;

(d) simple by $\pi_{1}$; or

(e) $\pi_{1}$ by simple by $\pi_{1}$.

From this Lemma we can deduce that if $G$ is neither Frobenius nor 2-Frobenius, then $G$ has a normal series $1 \triangleleft N \triangleleft M \triangleleft G$ such that $N$ is a nilpotent $\pi_{1}$-group, $M / N$ is a simple group and $G / M$ is a solvable $\pi_{1}$-group.

Lemma 2.4. Let $G$ be a finite group, $N$ a normal subgroup of $G$, and $G / N$ a Frobenius group with kernel $F$ and cyclic complement $C$. If $(|F|,|N|)=1$ and $F$ is not contained in $N C_{G}(N) / N$, then $p|C| \in \pi_{e}(G)$ for some prime divisor $p$ of $|N|$.

Proof. This follows from [8].

Lemma 2.5. Let $G$ be a simple group that satisfies property $P_{3}$. Then $G$ has one of the following structures:

(a) $L_{2}(q), q=2^{f}$ or $q=r^{f} \equiv 3,5(\bmod 8)$, where $\frac{q+1}{d}$ and $\frac{q-1}{d}$ are products of at most two primes, $d=(2, q-1)$;

(b) $L_{2}(9), L_{2}(7)$ or $L_{3}(4)$; or 
(c) $S z(q)$, where $q=2^{2 n+1}, n \geq 1, q-1, q-\sqrt{2 q}+1$ and $q+\sqrt{2 q}+1$ are products of at most two primes.

Proof. Let $P \in \operatorname{Syl}_{2}(G)$. If $P$ is non-abelian, then $4 \in \pi_{e}(G)$ and hence $\pi_{1}=$ $\{2\}$. It follows that $G$ is a $C_{22}$-group and therefore is isomorphic to $L_{2}(q), L_{3}(4)$ or $S z\left(2^{2 n+1}\right)(n \geq 1)$ by [9]. If $G \cong L_{2}(q)$, then $q \equiv 1$ or $7(\bmod 8)$. Observing that $(q+1) / 2,(q-1) / 2 \in \pi_{e}\left(L_{2}(q)\right)$, we conclude that if $q \equiv 1(\bmod 8)$, then $(q-1) / 2=4$ and $q=9$, so $G \cong L_{2}(9)$; if $q \equiv 7(\bmod 8)$, then $(q+1) / 2=4$ and $q=7$, so $G \cong L_{2}(7)$.

If $P$ is abelian, we conclude from [10, Ch. XI, Theorem 13.7] that $G \cong L_{2}(q)$, $q=2^{f}$ or $q \equiv 3$ or $5(\bmod 8), J_{1}$, or $R(q)$. Since $\left|\pi_{1}\left(J_{1}\right)\right| \geq 3$ (see [11]) and $\left|\pi_{1}(R(q))\right| \geq 3$ (see [10, Ch. XI, Theorem 13.4]), we have $G \cong L_{2}(q)$.

Considering the cyclic subgroups in $L_{2}(q)$ and $S z(q)$, we can obtain other properties of $G$.

Lemma 2.6. Suppose that $G$ is a finite group with property $P_{n}$. If there exists an element $x \in G$ whose order is a product of $m$ distinct primes, then $2^{m-1}+1 \leq n$.

Proof. Let $x_{1} \in\langle x\rangle$ be of order a prime $p_{1}$. Then there are $2^{m-1}$ elements $x_{1} y_{i}$ of distinct orders where $y_{i} \in\left\langle x^{p_{1}}\right\rangle$. For those $2^{m-1}$ elements, their orders have the common divisor $p_{1}$. This implies that $2^{m-1}+1 \leq n$.

3. Main results. Now, we are ready to formulate our results.

Theorem 3.1. Let $G$ be a finite solvable group that satisfies $P_{3}$ and $G_{p} \in$ $\operatorname{Syl}_{p}(G)$. Then one of the following statements holds.

(a) $G$ is a p-group with $\exp (G) \leq p^{2}$.

(b) $G=G_{p} G_{q}$ with $\exp \left(G_{p}\right)=p$ and $\exp \left(G_{q}\right)=q$.

(c) $G$ is a Frobenius group with kernel $N$ and complement $H$, where $N=G_{p}$ with $\exp (N) \leq p^{2}$ or $N=G_{p} \times G_{q}$ with $\exp \left(G_{p}\right)=p$ and $\exp \left(G_{q}\right)=q$; while $H$ is isomorphic to $Q_{8}$ or a subgroup of $Z_{r s}, r, s$ primes.

(d) $G$ is 2-Frobenius, that is $G$ has a normal series $1 \triangleleft N \triangleleft K \triangleleft G$ such that $G / N$ and $K$ are Frobenius groups with kernels $K / N$ and $N$ respectively, where $K / N$ is isomorphic to a subgroup of $Z_{r s}, r, s$ primes and $G / K$ is of order $p$; while $N$ is a q-group with $\exp (N)=q$ or a p-group with $\exp \left(G_{p}\right) \leq p^{2}$.

Proof. If $t(G)=1$, then $|\pi(G)| \leq 2$. We conclude that $G$ is the group in (a) or (b). If $t(G) \geq 2$, since $G$ is solvable, we conclude from Lemma 2.3 that $t(G)=2$ and $G$ is Frobenius or 2-Frobenius.

Suppose first that $G$ is a Frobenius group with kernel $N$ and complement $H$. We have $\pi_{1}=\pi(H)$ and $\pi_{2}=\pi(N)$ and thus $\left|\pi_{i}\right| \leq 2$ for $i=1,2$. Since $N$ is nilpotent, we conclude that $N=G_{p}$ with $\exp (N) \leq p^{2}$ or $N=G_{p} \times G_{q}$ with $\exp (P)=p$ and $\exp (Q)=q$. If $\pi(H)=\{r\}$, then $H$ is isomorphic to $Q_{8}$ or a subgroup of $Z_{r^{2}}$; if $|\pi(H)|=2$, then $H$ is a product of two Sylow subgroups of prime orders of $G$. The statement (c) holds.

Suppose now that $G$ is 2-Frobenius. Then $G$ has a normal series $1 \triangleleft N \triangleleft$ $K \triangleleft G$ such that $G / N$ and $K$ are Frobenius groups with kernels $K / N$ and $N$ 
respectively. It follows that $\pi_{1}=\pi(G / K) \cup \pi(N)$ and $K / N$ is a cyclic $\pi_{2}$-group with $\left|\pi_{i}\right| \leq 2$ for $i=1,2$. If $\pi(G / K)=\{p, q\}$, then $p$ or $q$ belongs to $\pi(N)$. We may assume without loss of generality that $q \in \pi(N)$. If $p=2$ and the Sylow 2-subgroups of $G / K$ are generalized quaternion groups, we conclude that 2,4 and $2 q \in \pi_{e}(G)$, a contradiction. It follows that $G / K$ is a cyclic group of order $p q$. Since $N$ is nilpotent, by induction we may assume that $N$ is an elementary abelian $q$-group. We conclude from Lemma 2.4 that $p q^{2} \in \pi_{e}(G)$, a contradiction. So $G / K$ is a $p$-group and $|\pi(N)|=1$. We have that if $\pi(N)=$ $\{q\}$ and $q \neq p$, then $N$ is a $q$-group with $\exp (N)=q$; if $\pi(N)=\{p\}$, then $\exp \left(G_{p}\right) \leq p^{2}$. It is clear that $K / N$ is isomorphic to a subgroup of $Z_{r s}$ with $r, s \in \pi(G)$. The statement (d) holds.

Theorem 3.2. Let $G$ be a finite non-solvable group that satisfies $P_{3}$. Then one of the following statements holds.

(a) $G / O_{2}(G) \cong L_{2}(q)$, where $q=2^{f} \geq 4$ and $q+1, q-1$ are products of at most two primes.

(b) $G \cong L_{2}(q)$, where $q>5$ and $q \equiv 3$ or $5(\bmod 8), \frac{q+1}{2}$ and $\frac{q-1}{2}$ are products of at most two primes.

(c) $G \cong L_{2}(7), L_{2}(9)$, or $L_{3}(4)$.

(d) $G \cong S z(q)$, where $q=2^{2 n+1}, n \geq 1, q-1, q-\sqrt{2 q}+1$ and $q+\sqrt{2 q}+1$ are products of at most two primes.

Proof. Since $G$ is unsolvable, we conclude that $|\pi(G)| \geq 3$ and $t(G) \geq 2$. By Lemma 2.3, $G$ has a normal series $1 \triangleleft N \triangleleft M \triangleleft G$ such that $N$ is a nilpotent $\pi_{1}$-group, $M / N$ is one of the simple groups listed in Lemma 2.5 and $G / M$ is a solvable $\pi_{1}$-group.

Claim 1. $G=M$.

Suppose first that $M / N \cong L_{2}(7), L_{2}(9)$ or $L_{3}(4)$. We conclude from [11] that $G=M$.

Suppose now that $q=r^{f} \equiv 3$ or $5(\bmod 8)$ or $q=2^{f}$. Let $t \in \pi(G / M)$. Since $G / M \leq \operatorname{Out}(M / N)$, we have $t|| \operatorname{Out}(M / N) \mid$. Observe that $|\operatorname{Out}(M / N)|=f d$, where $d=(q-1,2)$, it follows that if $t \wedge f$ then $t=d=2$ and $q \equiv 3$ or $5(\bmod 8)$. This forces $G / N \cong P G L(2, q)$, which implies that $G / N$ has at least three elements of distinct even orders, a contradiction. Thus $t \mid f$.

If $q \equiv 3(\bmod 8)$, then $\frac{q+1}{2}=2 t_{1}$ and $t_{1}$ is an odd prime. We conclude from Lemma 2.2 that $f=t$ is an odd prime, which implies that $t=t_{1}$ and so that $\frac{q+1}{2}=\frac{1}{2}\left(r^{t}+1\right)=2 t$, a contradiction. If $q \equiv 5(\bmod 8)$, a similar argument yields a contradiction. If $q=2^{f}$, we also conclude from Lemma 2.2 that $f=t$ is an odd prime (note that $L_{2}(4) \cong L_{2}(5)$, the case of $q=5$ has been considered). Observe that $|G / M|=|\operatorname{Out}(M / N)|=f$, we claim that $f \wedge|M / N|$. If this is false, then $f \mid 2^{f}+1$ or $f \mid 2^{f}-1$. It follows that $f, 2 f, 2^{f}+1 \in \pi_{e}(G / N)$ or $f, 2 f, 2^{f}-1 \in \pi_{e}(G / N)$, a contradiction. Let $x \in G / N-M / N$ such that $x$ is of order $f$. We conclude that $x$ acts fixed-point freely on the subgroups of orders $2^{f}+1$ and $2^{f}-1$ of $M / N$ and therefore $f \mid\left(2^{f}+1\right)+1$ and $f \mid\left(2^{f}-1\right)+1$. This forces $f \mid 2$, a contradiction. 
Suppose then that $M / N \cong S z\left(2^{2 n+1}\right)$. We have $\mid$ Out $(M / N) \mid=2 n+1$. If $G>M$, since $|G / M||| O u t(M / N) \mid$ and $\pi(G / M)=\pi_{1}(G)=\{2\}$, we have $2 \mid 2 n+1$, a contradiction.

Claim 2. $N$ is a normal $p$-subgroup of $G$ and $C_{G}(N) \leq N$. In particular, $N=O_{p}(G)$.

Since $N$ is a nilpotent $\pi_{1}$-group, by Lemma 2.1 we can assume that $N$ is a p-group. If $C_{G}(N) \not \leq N$, then $G=C_{G}(N) N$. We conclude that $G$ has elements of distinct orders $p, p l$ and $p m$ respectively, where $l, m \in \pi(G)$, a contradiction.

Claim 3. If $G / N \cong L_{2}(q), q=2^{f} \geq 4$, then $N$ is a 2-group. Otherwise $N=1$.

Suppose first that $G / N \cong L_{2}(q), q=2^{f} \geq 4$. Since $N$ is a $p$-group, we may assume from Lemma 2.1 that $N$ is a minimal normal subgroup of $G$. Let $P \in S l y_{2}(G / N)$, and $Q=N_{G / N}(P)$ and $W / N \cong Q$. If $N$ is not a 2-group, then $Q$ is a Frobenius group of order $\left(2^{f}-1\right) 2^{f}$. From Claim 2, we conclude that $Q$ acts faithfully on $N$. It follows from Lemma 2.4 that $\left(2^{f}-1\right) p \in \pi_{e}(W)$. Also, we have $2 p \in \pi_{e}(W)$ since $P$ is elementary abelian, a contradiction. Therefore $N$ is a 2-group.

Suppose now that $G / N \cong L_{2}(q)$, where $q=r^{f}>5$ and $q \equiv 3$ or $5(\bmod 8)$. Arguing as in the preceding paragraph, we conclude that $N=1$.

Suppose then that $G / N \cong L_{2}(9), L_{2}(7)$ or $L_{3}(4)$. In order to prove $N=1$, let $G$ be a minimal counterexample. We may assume that $N$ is a minimal normal subgroup of $G$. We conclude from the above argument that $\pi_{1}(G)=\{2\}$ and so $N$ is an elementary abelian 2-group. By claim 2, all $2^{\prime}$-subgroups of $G$ act fixed-point freely on $N$. If $G / N=L_{2}(9)$, since $G / N$ has Frobenius subgroups of order 36, we conclude from Lemma 2.4 that $8 \in \pi_{e}(W)$, a contradiction. If $G / N \cong L_{2}(7)$ or $L_{3}(4)$, a similar argument yields a contradiction.

Suppose finally that $G / N=S z(q)$, where $q=2^{2 n+1}, n \geq 1$. If $N>1$, we may assume that $N$ is an elementary abelian 2-group. Since $G / N$ has Frobenius subgroups of order $4(q-\sqrt{2 q}+1)$ with cyclic complements of order four, we conclude from Lemma 2.4 that $8 \in \pi_{e}(G)$, a contradiction.

Theorem 3.3. If $G$ is a finite group that satisfies $P_{n}$, then $|\pi(G)| \leq$ $C(\log n)^{4} \log \log n$, where $C$ is a constant. In particular, $\left|\pi_{e}(G)\right| \leq C(n-$ 1) $(\log n)^{4} \log \log n+1$.

Proof. Since $G$ satisfies $P_{n}$, we conclude that $\left|\pi_{e}(G)\right| \leq(n-1)|\pi(G)|+1$. By Lemma 2.6 we have that the number of different prime divisors of the order of an element of $G$ is bounded by a logarithmic function of $n$. The result follows from Theorem A of [12].

\section{ACKNOWLEDGEMENT}

This work has been supported by the National Natural Science Foundation of China (grant No. 10671026), and by the Scientific Research Fund of Hunan Provincial Education Department, and by the Scientific Research Fund of Changsha University of Science and Technology. 


\section{REFERENCES}

1. D. Benjamin, Coprimeness among irreducible character degrees of finite solvable groups. Proc. Amer. Math. Soc. 125(1997), No. 10, 2831-2837.

2. J. K. MCVey, Prime divisibility among degrees of solvable groups. Comm. Algebra 32(2004), No. 9, 3391-3402.

3. A. Moretó, G. Qian, and W. Shi, Finite groups whose conjugacy class graphs have few vertices. Arch. Math. (Basel) 85(2005), No. 2, 101-107.

4. M. FANG and P. ZHANG, Finite groups with graphs containing no triangles. J. Algebra 264(2003), No. 2, 613-619.

5. W. SHI, A new characterization of $A_{5}$ and finite groups with all elements of prime order. (Chinese) J. Southwest China Normal University 8(1984), No. 1, 36-40.

6. M. Deaconescu, Classification of finite groups with all elements of prime order. Proc. Amer. Math. Soc. 106(1989), No. 3, 625-629; K. N. Cheng, M. Deaconescu, M.-L. Lang, and W. J. Shi, Corrigendum and addendum to: "Classification of finite groups with all elements of prime order" [Proc. Amer. Math. Soc. 106 (1989), No. 3, 625-629; MR0969518 (89k:20038)] by Deaconescu. Proc. Amer. Math. Soc. 117(1993), No. 4, 1205-1207.

7. J. S. Williams, Prime graph components of finite groups. J. Algebra 69(1981), No. 2, 487-513.

8. V. D. Mazurov, Characterizations of finite groups by sets of the orders of their elements. (Russian) Algebra i Logika 36(1997), No. 1, 37-53; English transl.: Algebra and Logic 36(1997), No. 1, 23-32.

9. M. Suzuki, Finite groups with nilpotent centralizers. Trans. Amer. Math. Soc. 99(1961), 425-470.

10. B. Huppert and N. BlackBurn, Finite groups. III. Grundlehren der Mathematischen Wissenschaften [Fundamental Principles of Mathematical Sciences], 243. SpringerVerlag, Berlin-New York, 1982.

11. J. H. Conway, R. T. Curtis, S. P. Norton, R. A. Parker, and R. A. Wilson, Atlas of finite groups. Maximal subgroups and ordinary characters for simple groups. Oxford University Press, Eynsham, 1985.

12. A. Moretó, On the number of different prime divisors of element orders. Proc. Amer. Math. Soc. 134(2006), No. 3, 617-619 (electronic).

(Received 22.03.2006)

Author's address:

College of Mathematics and Computing

Changsha University of Science and Technology

Changsha, Hunan, 410077

P. R. China

E-mail: xzyou2003@yahoo.com.cn 